through estimating the potential impact and cost of these interventions across LAC.

Methods WHO estimates of the potential impact of three interventions in four Latin American countries were extrapolated, based on UN estimates of population size and for the years 2005-2010, to all areas of LAC.

Results A 15\% reduction in salt intake (628 000 deaths averted), 25\% reduction in smoking through increased implementation of the Framework Convention on Tobacco Control (441500) deaths averted), and scaling up treatment of $60 \%$ those already in contact with health services at high risk of cardiovascular death (1 167000 deaths averted), would avert a total of 2.2 million deaths in LAC over 10 years. More than half of these deaths would be in people aged $<70$ years old. These interventions would cost $<1 \%$ of the existing health budget.

Conclusion These estimates show that substantial benefits are achievable, and are being used to prioritise activities within the LAC region.

\section{P2-265 MORTALITY IN OLDER MEN OF SOUTHEAST BRAZIL: A STUDY OF ASSOCIATED CAUSES OF DEATH}

doi:10.1136/jech.2011.142976j.98

L M Santiago, ${ }^{*}$ L L Luz, I E Mattos. Oswaldo Cruz Foundation, National School of Public Health, Rio de Janeiro, Rio de Janeiro, Brazil

Introduction The adoption of unhealthy western lifestyles has contributed to changes in mortality patterns in developing countries. The aim of this study was to evaluate associated causes of death in older men in a medium-size Brazilian city.

Methods The study was based on a cohort of 2859 older men living in Juiz de Fora, Brazil, followed from 2006 to 2010. All death certificates were retrieved from the Mortality Information System of the city. Underlying and associated causes of death were coded according to the 10th International Classification of Diseases.

Results There were 298 deaths and mean age at death was 73.4 years. Diseases of the circulatory system (I00-199) corresponded to 108 (36.2\%) deaths and its major associated causes were other circulatory diseases, diseases of the respiratory system (J00-J99) and infectious and parasitic diseases (A00-B99). Neoplasms corresponded to 65 deaths $(21.8 \%)$ and predominant associated causes were respiratory diseases, mainly pneumonias, and infectious and parasitic diseases. Diseases of the respiratory system corresponded to $44(14.8 \%)$ deaths and infectious and parasitic diseases were the main associated cause. These three groups comprised $72.8 \%$ of the underlying causes in the cohort.

Conclusion Circulatory and respiratory diseases and neoplasms were major causes of mortality in this cohort and also represent an important public health problem in Brazil. The influence of a westernised life style is probably reflected in this pattern of chronic diseases. Although not as important, infectious and parasitic diseases are still present as associated causes and likely worsening health conditions in this cohort.

\section{P2-266 RELATIVE VS CANCER-SPECIFIC SURVIVAL: ASSUMPTIONS AND POTENTIAL BIAS}

doi:10.1136/jech.2011.142976j.99

${ }^{1} \mathrm{D}$ Sarfati, ${ }^{*} \mathrm{~T}$ Blakely, ${ }^{1} \mathrm{M}$ Soeberg, ${ }^{1} \mathrm{~K}$ Carter, ${ }^{2} \mathrm{~N}$ Pearce. ${ }^{1}$ University of Otago, Wellington, New Zealand; ${ }^{2}$ Massey University, Wellington, New Zealand

Introduction Cancer-specific and relative survival analyses are the two main methods of estimating net cancer survival. Bias through misclassification of cause of death is well recognised for cancerspecific survival, but to date there has been no systematic examination of the potential bias from using an external comparison group for relative survival. This latter bias may be particularly important for smoking-related cancers where the expected survival is lower than the general population because of the high incidence of non-cancer smoking-related mortality.

Methods We use unique data from the New Zealand census which provides information on individual smoking status, allowing us to produce smoking-adjusted life tables. We apply these to relative survival estimates for lung and bladder cancers, known to be strongly associated with smoking. We also compare these with simulations to estimate the effect of misclassification bias on cancerspecific estimates.

Results Five-year relative survival estimates were similar regardless of which life tables were used for lung cancer. For bladder cancer, estimates varied more markedly; for example, 5-year survival estimates ranged between 0.70 and 0.84 for male smokers. Simulations suggested that for cancer-specific analyses, bias of up to $20 \%$ misclassification of cause of death resulted in estimates of 5 -year survival that were $1.3-19 \%$ different from "true" estimates, with largest error for those cancers with poorest survival.

Conclusions Both cancer-specific and relative survival methods are potentially valid for population-based cancer survival studies. Both methods are susceptible to bias which is sensitive to the survival probability of the cancer under study.

\section{P2-267 THE UNUSUAL EPIDEMIOLOGY OF TESTICULAR CANCER IN NEW ZEALAND}

doi:10.1136/jech.2011.142976j.100

D Sarfati, ${ }^{*}$ C Shaw, T Blakely, J Atkinson, J Stanley. University of Otago, Wellington New Zealand

Introduction Testicular cancer (TC) is increasing rapidly in developed countries. Drivers of these trends remain obscure. Ethnic differences in TC incidence within countries are often marked; white populations consistently having the highest rates. Many studies have found that high socioeconomic status is a risk factor for TC. There is some evidence that epidemiological patterns of TC may differ in New Zealand. This study investigates the ethnic and socioeconomic patterns of TC incidence in New Zealand.

Methods Cohorts of the entire New Zealand population for 1981-1986, 1986-1991, 1991-1996, 1996-2001, 2001-2004 were created, and probabilistically linked to cancer registry records, allowing direct determination of ethnic and household income incidence and trends in TC.

Results The study included 2000 cases of TC. We found increasing rates of TC for all ethnic and income groups since the 1990s. Māori had higher rates, and Pacific and Asian lower rates than European men with rate ratios pooled over time of 1.51 (95\% CI 1.31 to 1.74 ); 0.40 (95\% CI 0.26 to 0.61$)$ and 0.54 (95\% CI 0.31 to 0.94$)$ respectively. Men with low incomes had higher risk of TC than those with high incomes (pooled rate ratio for lowest to highest income groups $=1.23 ; 95 \%$ CI 1.05 to 1.44 ).

Conclusions New Zealand is unique in the world, having the only non-white population with a higher TC incidence than the local white population. Also unusually, lower socioeconomic men have higher rates of TC. Given the lack of understanding of TC aetiology, these unusual patterns may provide clues for future research. 